\title{
Traceability of dynamic magnetic fields by NMR magnetometry
}

\author{
R. V. Martin ${ }^{1}$, D. J. Nazarre ${ }^{1}$, E. A. Périgo ${ }^{2}$ \\ ${ }^{1}$ Institute for Technological Research, São Paulo, SP 05508-901 Brazil \\ ramon@ipt.br \\ ${ }^{2}$ Laboratory for the Physics of Advanced Materials, University of Luxembourg, Luxembourg, L1511 Luxembourg
}

\begin{abstract}
A method to provide traceability with low uncertainties for magnetic alternating fields is presented. The reconstruction of an $\mathrm{AC}$ field waveform is performed by the measurement of the NMR pulses time position obtained by the superposition of a static magnetic field to the AC magnetic field. A frequency sweep in the excitation of the NMR probe allows the changing of the pulses positions covering all the $\mathrm{AC}$ waveform. As the frequency at which the NMR pulse exists is related only to the proton gyromagnetic ratio, it can be considered as a primary standard. A prototype tested experimentally shows the feasibility of the method proposed.
\end{abstract}

Index Terms - Magnetic measurements, magnetic standards, dynamic fields, AC magnetic fields.

\section{INTRODUCTION}

The use of nuclear magnetic resonance (NMR) magnetometry made possible the reduction of the static magnetic fields relative uncertainty to the order of $10^{-6}$, although it is still much larger for AC magnetic signals [1]. In general, Faraday's induction methods are employed for the latter [2]. The measurement of the dynamic field intensity is made indirectly from the integration of the induced voltage signal in pick-up coils with $N$ turns due to magnetic flux variation $d \varnothing / d t[2]$ :

$$
V=-N \frac{d \phi}{d t}
$$

In (1), assuming a sinusoidal signal $V=-N A w \cos w t$, one may write:

$$
B_{0} \sin w t=\frac{\int v d t}{N A},
$$

where $A$ represents the area of the coil.

The uncertainty of ( $\left.B_{0} \sin w t\right)$ arises either from the calibration factor of the fluxmeter that performs the integration or from the determination of the constant $N A$ of the pick-up coil (total uncertainty $U_{T}>1 \%$ ). Another difficulty existent in induction methods is the susceptibility to high frequency noise and the signal-to-noise degradation for low frequencies: $w \rightarrow 0 \Rightarrow V \rightarrow 0$. In order to overcome these issues an approach based on NMR magnetometry is addressed.

\section{PRINCIPLE OF AC NMR MAGNETOMETRY}

NMR magnetometry makes use of the proton magnetic resonance expressed by:

$$
f=G_{p} \cdot B
$$

where $f$ is the frequency, $G_{p}$ represents the proton gyromagnetic factor $\left(42.57608(12) \mathrm{MHzT}^{-1}[3]\right)$, and $B$ is the magnetic induction.

The protons absorb energy when an external excitation field is applied with this frequency. Such an effect is detected by measuring the perturbation in the quality factor $Q$ of a water filled coil in the NMR probe head. To extend the use of NMR for AC fields, the hardware is similar to the one used in DC. A static polarization field $B_{D C}$ should be added to the AC field $B_{A C}$ because the system is only sensitive to the module of the field. This static field should be adjusted in order to maintain all the waveform points with the same polarity and with a minimum level for detection so that $B_{T}=\left(B_{A C} B_{D C}\right)>0$. Figure 1 shows the principle of the method for AC traceability. The interval time $t_{1}, t_{2}$ and $t_{3}$ and the frequency $f_{e x}$ are the parameters used to reconstruct the waveform by means of sampling techniques.

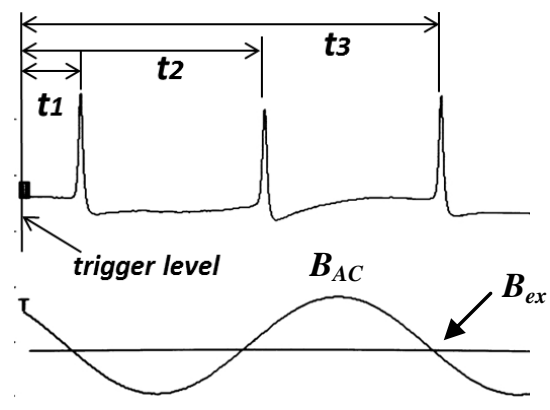

Fig. 1. NMR pulses observed when the $B_{T}$ waveform crosses an excitation level: $B_{G x}=f_{G x} / G_{P}$.

\section{EXPERIMENTAL SETUP}

In order to evaluate the proposed methodology a commercial NMR teslameter (Metrolab PT2025) was modified by adding an external RF source to generate the sweeping of the excitation frequency $f_{e x}$. The NMR signal output was connected to a data acquisition system to measure $t_{1}, t_{2}$ and $t_{3}$. The field $B_{D C}$ was generated with a commercial electromagnet (Laborattorio Eletrofisico IS-300) and the field $B_{A C}$ was produced with an auxiliary coil wrapped around the yoke. Due the technical limitations of the NMR teslameter a static polarization field around $100 \mathrm{mT}$ was used. Figure 2 shows the schematic diagram of the hardware. 


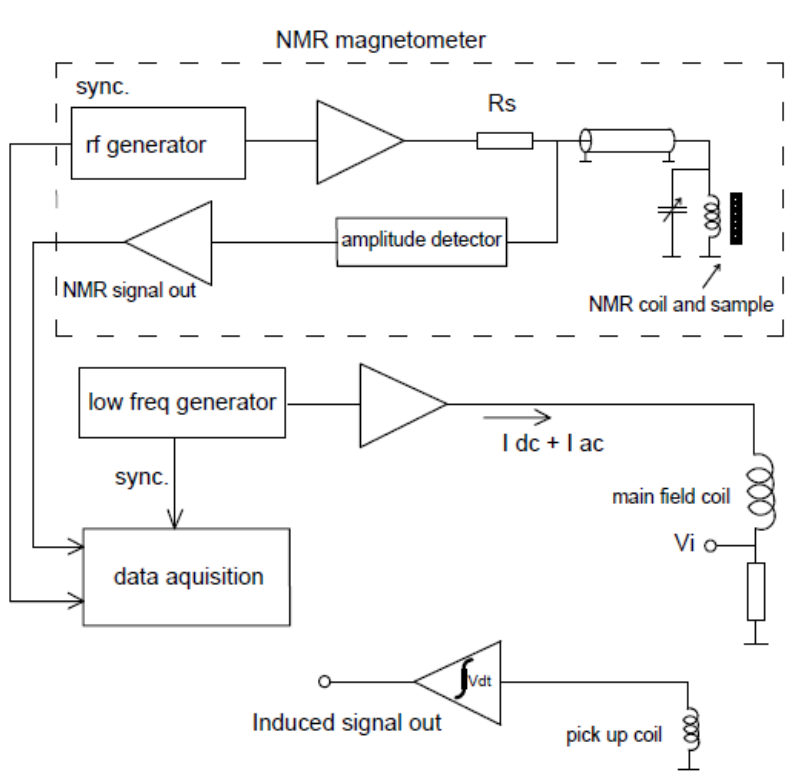

Fig. 2. A simplified diagram of the NMR system used to provide AC traceability.

\section{RESULTS AND DISCUSSION}

The system allows the reconstruction of arbitrary waveforms from DC up to $1 \mathrm{kHz}$. Figure 3 depicts a typical example of the NMR signal for few excitation levels and an AC sinusoidal field with $480 \mu \mathrm{T}_{\mathrm{rms}}, 60 \mathrm{~Hz}$. Figure 4 shows the reconstructed waveform from the simultaneously measurement of $t_{1}, t_{2}, t_{3}$ and $f_{e x}$. The magnetic and electrical noise shift the peak position, but this noise can be cancelled (asynchronous noise) by averaging the NMR pulses if a trigger signal is available.
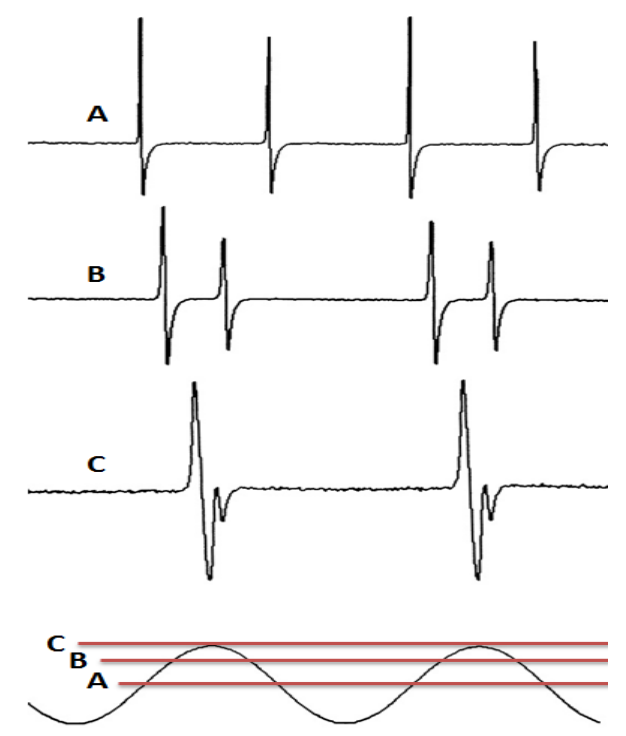

Fig. 3. Arrangement of few experimental pulses resulting from different excitation levels along the frequency sweep.

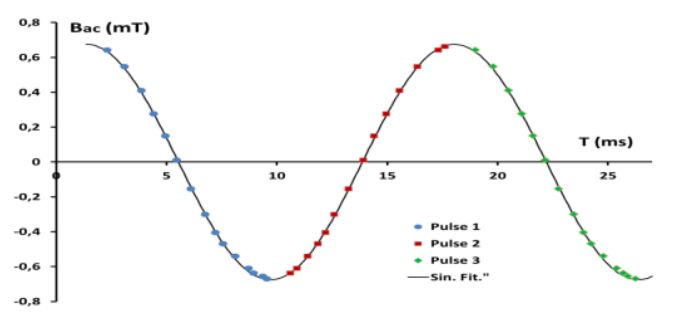

Fig. 4. Waveform reconstructed from NMR measured data of fig. 3.

The main contribution of the uncertainty in NMR magnetometry comes from the measurement of the pulses position. Therefore, it is highly desirable to have short ones. The pulse width shrinks with better field homogeneity and stability, lower noise, and reducing the spin-lattice relaxation time $T_{l}$. This is a property of the sample inside NMR probe coil. It can be improved changing the material or adding some paramagnetic salt in the water of the NMR sample. It was possible to observe, as shown in Figure 5, that the relative pulse width also is proportional to the waveform derivative and to the ratio of the bias field intensity and the amplitude of the AC field: $B_{D C} / B_{A C}$.

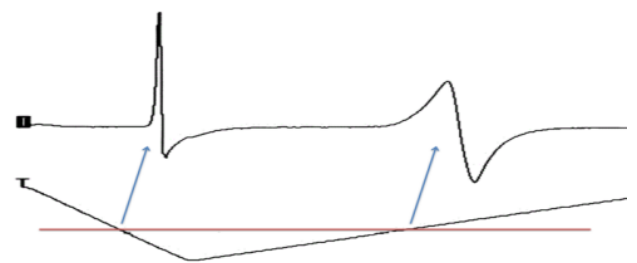

Fig. 5. Experimental NMR pulse shape observed as a function of the waveform derivative.

\section{INTERIM CONCLUSIONS}

NMR magnetometry presents a real potential for obtaining primary traceability of magnetic quantities. A further development is necessary to shrink the NMR pulses and to extend the dynamic range to allow the measurement of higher harmonics of the field waveform. Further discussions will be carried out in the full manuscript.

\section{ACKNOWLEDGEMENTS}

The authors thank FINEP/CNPq for the financial support of this investigation by means of the SIBRATEC program.

\section{REFERENCES}

[1] K. Weyand, "Maintenance and Dissemination of the Magnetic Field Unit at PTB", IEEE I-M, 50, 2, pp 470-473, April 2001.

[2] R. V. Martin, D. J. Nazarre and R. R. Dias, "Rastreabilidade para campos magnéticos alternados até $10 \mathrm{kHz}$ ", VII Metrologia proceedings, Ouro Preto - MG, Nov. 2013.

[3] Gp by CODATA at NIST: http://physics.nist.gov/cgibin/cuu/Value?gammapbar/search for=atomnuc!). 